Itinéraires Itinéraires

Littérature, textes, cultures

\title{
L'ironie et le genre : spécificité du héros dans le roman occitan de Jaufré
}

Andrea Valentini

\section{OpenEdition}

\section{Journals}

Édition électronique

URL : http://journals.openedition.org/itineraires/2203

DOI : $10.4000 /$ itineraires.2203

ISSN : 2427-920X

Éditeur

Pléiade

\section{Édition imprimée}

Date de publication : 1 décembre 2008

Pagination : 35-47

ISBN : 978-2-296-07519-1

ISSN : 2100-1340

\section{Référence électronique}

Andrea Valentini, «L'ironie et le genre : spécificité du héros dans le roman occitan de Jaufré », Itinéraires [En ligne], Numéro inaugural | 2008, mis en ligne le 01 décembre 2008, consulté le 19 avril 2019. URL : http://journals.openedition.org/itineraires/2203 ; DOI : 10.4000/itineraires.2203

Licence Creative Commons

Itinéraires est mis à disposition selon les termes de la licence Creative Commons Attribution - Pas d'Utilisation Commerciale - Pas de Modification 4.0 International. 


\title{
L'ironie et le genre: spécificité du héros dans le roman occitan de Jaufré
}

\begin{abstract}
Jaufré is the only surviving Arthurian romance in Occitan. Writing probably in the first quarter of the thirteenth century, its author no longer adhered unquestioningly to Arthurian values, which he regarded with a certain irony. As a result, the poem's two main characters are distinguished by unusual features: Jaufré often admits to being afraid and displays traits such as fickleness, more typical of youth than of manhood; unlike more traditional heroines, Brunissen is at times tyrannical and headstrong. Through a series of comparisons and literary analyses, this essay examines how the genre of Jaufré and its representation of gender are intimately related.
\end{abstract}

\section{Introduction}

Le Jaufré est le seul roman arthurien en vers conservé en langue d'oc'. Il ne nous appartient pas ici d'essayer de trancher la question de savoir si ce roman est le fruit d'un seul ou de deux auteurs, et il nous suffira de rappeler que les savants qui penchent pour la première solution sont plus nombreux ${ }^{2}$. Les opinions divergent également quant à la date de composition du roman: l'hypothèse la plus vraisemblable semble être celle qui la place sous le règne de Jacques I ${ }^{\text {er }} \mathrm{d}^{\prime}$ Aragon (1213-1276) ${ }^{3}$.

1. Sur la littérature romanesque en langue d'oc, voir E. Baumgartner, «Le Roman aux XII ${ }^{\mathrm{e}}$ et XIII ${ }^{\mathrm{e}}$ siècles dans la littérature occitane", dans Le Roman jusqu'à la fin du XIII siècle, dir. J. Frappier et R. R. Grimm, Grundriss der romanischen Literaturen des Mittelalters, vol. IV, t. I, Heidelberg, Carl Winter-Universitätsverlag, 1978, p. 627-644.

2. Aux v. 10945-10954 on mentionne « cel que-1 romantz comenset » (celui qui commença le roman) et "aquel que l'acabet» (celui qui l'acheva). Pour une revue de l'abondante bibliographie sur cette question, voir Jaufre, éd. et trad. italienne C. Lee, Rome, Carocci, 2006, p. 35-42.

3. Le roman est dédié à un roi d'Aragon (v. 61-84 et 2622-2630). Les deux autres rois 
Nous ne pouvons pas non plus résumer l'intrigue dans le détail et nous nous en tiendrons à l'essentiel: nouvellement adoubé, Jaufré part en quête de Taulat de Rougemont, qui a tué un chevalier, sans le défier, devant la reine (ici Guilalmier et non pas Guenièvre). Après une suite d'aventures, Jaufré arrive à vaincre Taulat vers le milieu du roman. Entre-temps, il a fait la connaissance de la belle Brunissen: dans la deuxième partie, les deux jeunes gens se dévoilent leur amour. Après d'autres aventures, Jaufré et Brunissen se marient en présence du roi Arthur, avant de rentrer à Monbrun, le fief de Brunissen.

Dans le présent article, nous nous intéresserons à la construction de la masculinité du héros, c'est-à-dire à son devenir homme et chevalier. Si d'une part le personnage principal présente certains caractères qu'on trouve partout ailleurs dans les romans arthuriens en vers - tels que la jeunesse vigoureuse, le courage, la générosité -, nous verrons, d'autre part, que le héros du Jaufré est en partie atypique; parallèlement, le personnage de Brunissen présente aussi des singularités. L'ironie du narrateur est à la base, croyons-nous, de ces traits étranges: l'auteur n'a peut-être pas, ou pas toujours, réfléchi à l'invraisemblance des aventures qu'il relate (le Jaufré ne présente pas de réflexions métadiscursives, et il ne peut pas être considéré comme un ancêtre du Don Quichotte, si ce n'est à l'état embryonnaire) ; le narrateur, toutefois, donne l'impression de ne plus adhérer à sa matière, et pour cela même il en exagère l'invraisemblance jusqu'à provoquer un sourire, ou à en rire lui-même, comme le soulignait déjà Clovis Brunel ${ }^{4}$. Aussi pouvons-nous affirmer que la construction du genre

auxquels ont pensé les interprètes sont Alphonse II (1162-1196) et Pierre II (1196-1213). G. Paris s'était déjà prononcé pour l'identification avec Jacques ${ }^{\text {er }}$ (dans la notice « Jaufré » de l'Histoire littéraire de la France, t. XXX, Paris, Imprimerie nationale, 1888, p. 215-217); son opinion a été acceptée par des savants tels que A. Jeanroy («Le Roman de Jaufré », Annales du Midi, n 53, 1941, p. 363-390 [p. 364-365]), C. Brunel (dans son édition critique, Jaufré. Roman arthurien du XIII siècle en vers provençaux, 2 vol., Paris, Société des Anciens Textes Français, 1943, vol. I, p. XxXVII-XL) et M. Zink (dans l'introduction de la traduction en français qu'il a fournie de la moitié du roman environ dans D. RégnierBohler, dir., La Légende arthurienne: Le Graal et la Table ronde, Paris, Laffont, 1989, p. 841-922 [p. 844]). Sur cette question, on trouvera une mise au point très claire, bien que déjà ancienne, dans P. Rémy, "À propos de la datation du Roman de Jaufré », Revue belge de philologie et d'histoire, $\mathrm{n}^{\circ} 28,1950$, p. 1349-1377.

4. Jaufré, éd. Brunel, vol. I, p. vIII. Le caractère de « discrète parodie » du roman est une des raisons qui déconseillent de penser à une date trop haute pour sa composition, comme le faisait remarquer Baumgartner, op. cit., p. 634. D'autres savants ont vu dans le Jaufré quelques aspects plus ou moins parodiques et/ou ironiques: entre autres H. R. Jauss, « Le Sens de l'aventure dans le roman de Jaufre ", Actes et Mémoires du $1^{\text {er }}$ Congrès international de langue et littérature du midi de la France, Avignon, Palais du Roure, 1957, p. 34-39 (p. 35); A. Limentani, L'eccezione narrativa. La Provenza medievale e l'arte del racconto, Turin, Einaudi, 1977, p. 92-101; B. Schmolke-Hasselmann, The Evolution of Arthurian Romance: The Verse Tradition from Chrétien to Froissart, trad. M. et R. Middleton, Cambridge, Cambridge University Press, 1998 (éd. originale allemande 
sexué d'un personnage de fiction n'est pas sans liens avec le genre littéraire de l'œuvre dans laquelle ce personnage apparaît et avec l'évolution de ce genre, comme Simon Gaunt l'a bien montré pour le domaine de la littérature médiévale ${ }^{5}$. Le point de vue que nous adopterons sera synchronique et non pas diachronique, l'œuvre littéraire étant ici analysée en elle-même et non pas philologiquement; pour interpréter quelques épisodes, nous utiliserons des classiques de l'anthropologie, de la psychanalyse et de la gender theory, là où il nous semblera qu'ils pourront étayer nos thèses.

\section{Le personnage de Jaufré}

Voici la première présentation du héros, lors de son arrivée à la cour du roi Arthur:
$\mathrm{Ab}$ aitan il viron intrar,
Cavalcan un rosin liar,
Un donzel gran, e bel e gen,
E venc molt içarnidament.

(v. 523-526)

C'est alors qu'on vit entrer, monté sur un roussin gris, un garçon grand, beau et gracieux, qui s'avança avec beaucoup d'aisance ${ }^{6}$.

Déjà cette entrée, où l'on met l'accent surtout sur la grâce du héros, qui néanmoins monte un « rosin », un cheval de peu de valeur, présente Jaufré sous une lumière particulière; le fait de souligner sa beauté, plutôt que sa vaillance ou sa force physique (ou mieux plutôt que de souligner sa beauté et ses vertus chevaleresques ${ }^{7}$ ), n'est peut-être pas anodin. En tout cas, deux aspects sont évidents: la jeunesse du héros et une certaine iro-

1980), p. 19; S. Fleischmann, «Jaufre or Chivalric Askew: Social Overtones of Parody in Arthurian Romance », Viator, n 12, 1981, p. 101-129; W. Calin, «Vers une nouvelle lecture de Jaufré: un dialogue avec Marc-René Jung », Marche romane, n 33, 1983, p. 3947 (p. 45); V. Fraser, « Humour and Satire in the Romance of Jaufre », Forum for Modern Language Studies, no 31, 1995, p. 223-233.

5. S. Gaunt, Gender and Genre in Medieval French Literature, Cambridge, Cambridge University Press, 1995.

6. Nous citons, aussi bien pour le texte occitan (avec quelques ajustements graphiques) que pour la traduction en français (que nous retouchons très légèrement), des Troubadours, éd. et trad. R. Lavaud et R. Nelli, 2 vol., Paris, Desclée de Brouwer, 1960-1966, vol. I, le Jaufré, p. 15-618. Celle-ci est l'édition d'accès le plus facile, mais il ne s'agit pas d'une édition critique à proprement parler: tout en introduisant quelques rares corrections, les auteurs prennent pour base l'édition de Brunel citée supra.

7. Par exemple, dans le Bel Inconnu de Renaud de Beaujeu ou de Bâgé, on lit à propos de Guinglain, quand il arrive à la cour d'Arthur, d'ailleurs sur un destrier et tout armé, qu'il «Bien sanbla chevalier a droit» (v. 76) et que «Molt i avoit biel baceler» (v. 96), éd. M. Perret, trad. M. Perret et I. Weil, Paris, Champion, 2003. 
nie du narrateur ${ }^{8}$ (nous pensons également à l'insistance sur la « gonela » [tunique], les « causas » [chausses] et la « garlanda » [couronne de fleurs] dans la description qu'on lit peu après [v. 539-544]).

Aussitôt adoubé, Jaufré se met en route à la recherche de Taulat de Rougemont, malgré les résistances du roi et les moqueries du sénéchal Queu ${ }^{9}$. Mais c'est un étrange chevalier que le nôtre: à plusieurs reprises, il est présenté comme un personnage apeuré, qui fuit devant des agresseurs. Dans ces cas-là, il ne renonce pas à ses devoirs, parce qu'il ne fuit que devant des ennemis qui ne sont pas eux-mêmes des chevaliers ou qui ne le défient pas, et avec lesquels il serait donc honteux de combattre : reste que certaines attitudes du héros sont incongrues.

Les habitants de la terre dont fait partie Monbrun, le fief de Brunissen, sont en deuil à cause d'un atroce supplice infligé à Mélian de Montmélior, leur seigneur, par Taulat de Rougemont, depuis que celui-ci l'a fait prisonnier. Les habitants se lèvent plusieurs fois par nuit pour se plaindre de cette captivité, et ils en souffrent tant qu'ils ne peuvent s'empêcher de battre tous ceux qui leur posent des questions sur ce sujet, comme Jaufré le découvrira plus tard (v. 4879-5169). Lors de son premier séjour au château de Monbrun, quand les barons qui l'ont en garde se lamentent au milieu de la nuit et que Jaufré leur en demande la cause, ceux-ci se jettent sur lui pour le frapper; enfoui sous les draps et protégé par son armure, Jaufré survit, mais « no·s vol moure de paor » (« ne veut pas bouger par crainte » [v. 3869]). Les deux enfants d'Augier d'Essart, qui avait promis à Jaufré de le traiter en fils en vertu de l'amitié qui l'avait lié à son père, se dressent sans ménagement contre le héros quand celui-ci les questionne à propos des lamentations, mais les « armes » qu'ils emploient à cette fin (un épervier et un lévrier!) nous font sourire, tout comme le héros qui s'enfuit sans rien dire (v. 4405-4413). Peu après, c'est Augier lui-même qui change d'attitude et qui qualifie Jaufré de « bastarz » (v. 4674), menaçant de le tuer, ce qui fait que le héros « pren a fugir » (« se met à fuir » [v. 4681]). Au sénéchal de Brunissen qui lui rappelle que, après que Jaufré eut vaincu les trois chevaliers qui l'avaient défié pour l'emmener à la châtelaine (voir ci-dessous),

8. Si la jeunesse est un caractère quasi constant des héros arthuriens, elle est en général la marque de leur vigueur: dans le Cligès de Chrétien de Troyes, par exemple, le héros n'a que quinze ou dix-sept ans, suivant les manuscrits, quand il réalise ses premiers exploits (éd. et trad. C. Mela et O. Collet, Paris, UGE, coll. « Livre de poche/Lettres gothiques », 1994, v. 2719).

9. Fidèle à son caractère de railleur, Queu, méprisant, dit à Jaufré qu'il ferait mieux de boire plutôt que de suivre Taulat (v. 604-612); à son retour à Carduel, Jaufré se vengera en vainquant le sénéchal en duel et en emmenant son cheval (v. 9436-9472). La malveillance de Queu ainsi que les incidents qui lui arrivent à la suite de ses railleries sont un leitmotiv de la littérature arthurienne que l'on retrouve déjà dans les romans de Chrétien de Troyes: voir, par exemple, dans l'Yvain les v. 588 sq., 2180 sq. et 2256 sq. (éd. et trad. D. Hult, Paris, UGE, coll. « Livre de poche/Lettres gothiques », 1994). 
tous les barons de la cour étaient venus le chercher en même temps, le héros confie avec candeur qu'il n'a jamais eu une peur aussi grande qu'à cette occasion (v. 7245-7246). Encore à la fin du roman, alors que Jaufré est un chevalier accompli, quand la fée de Gibel se présente à lui avec toute sa maisonnée pour lui offrir des cadeaux, il craint qu'elle ne vienne pour l'enlever avec ses enchantements, comme elle l'avait fait auparavant pour l'obliger à la protéger contre Félon d'Auberue, et il met en garde Mélian, suggérant qu'ils fassent armer leurs chevaliers (v. 10362-10365) ${ }^{10}$.

Si ces épisodes restent au stade d'un comique embryonnaire, un autre passage présente le héros sous une lumière franchement ridicule. Après avoir tué un redoutable lépreux géant qui s'apprêtait à violer une jeune fille, Jaufré est tellement apeuré et hors de lui qu'il frappe violemment celle-ci, croyant combattre encore contre son ennemi:

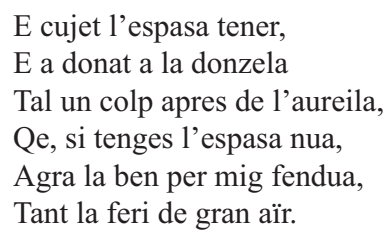

(v. 2462-2467)

Et croyant tenir son épée, il a donné à la jeune fille un coup si vif, près de l'oreille, que, s'il avait eu vraiment l'épée nue à la main, il l'aurait pourfendue par le milieu, tant il a mis de haine à frapper.

Un autre fait singulier dans le roman est l'inconstance de Jaufré. Lorsqu'il se met à la recherche de Taulat de Rougemont, il s'impose de ne pas manger et de ne pas dormir jusqu'à ce qu'il ait retrouvé son ennemi, engagement certes un peu bizarre même dans une œuvre de fiction ${ }^{11}$. Les premières aventures durant, il maintient cet engagement, mais, après le combat contre les lépreux (v. $\left.2180^{1}-3016\right)^{12}$, la nature prend le dessus :

10. Lorsque la fée, étonnée de voir les chevaliers tout armés, demandera des explications, Jaufré mentira: "Cujem nus ben, senes duptar,/ Fellonz fos que·s volgues venjar...» (« Nous croyions, sans aucun doute, que c'était Félon qui voulait se venger... » [v. 1044110442]).

11. Dans les romans arthuriens, il est rare que les héros se privent volontairement de manger; au contraire, ils ne se gênent pas pour le faire ou se plaignent souvent d'avoir faim: ainsi, entre autres, Gauvain dans Hunbaut ou Gligois et Méraugis de Portlesguez dans les romans homonymes.

12. Les exposants à la droite des numéros des vers ont été introduits par Brunel pour ne pas s'éloigner de la numérotation de l'édition critique qui avait précédé la sienne, procurée par H. Breuer, Jaufre. Ein altprovenzalischer Abenteuerroman des XIII. Jahrhunderts, Göttingen, Niemeyer, 1925. 
Tal son a c'ades va durmen

E ades sai e lai volven,

C'ades a paor de caser.

(v. 3031-3033)

Il a un tel sommeil que toujours il s'endort et toujours se laisse aller d'un côté ou de l'autre, et il a toujours peur de tomber [de cheval].

Ce passage, encore une fois, présente le héros sous une lumière vaguement humoristique et l'on aura remarqué qu'au vers 3033 on lit le mot «paor». Non seulement Jaufré est présenté comme un chevalier qui ne tient pas un engagement, mais encore c'est ce désir de dormir qui fait qu'il rencontre Brunissen: il finit en effet par s'endormir dans le jardin de Monbrun; les oiseaux, épouvantés par une présence inconnue, arrêtent leurs chants; la châtelaine, qui aime les écouter avant de se coucher, ordonne à ses barons d'aller vérifier ce qui se passe et, si quelqu'un est entré dans le jardin, de le lui amener pour qu'elle puisse le mettre à mort. Suit un épisode amusant, pendant lequel Jaufré, qui ne peut s'empêcher de dormir, est réveillé à trois reprises par trois chevaliers : il fait promettre aux deux premiers que, s'il les vainc, ils le laisseront dormir et, croyant qu'il s'agit toujours du même qui ne tient pas sa promesse, il blesse gravement le troisième; enfin, seule l'arrivée de tous les barons de la cour permettra à Brunissen d'avoir son prisonnier ${ }^{13}$.

Quand le héros se remet à poursuivre Taulat, il ne prend plus formellement l'engagement de ne pas s'arrêter, mais à plusieurs reprises il refuse, dans un premier temps, l'hospitalité des gens qu'il rencontre; ce qui est curieux, c'est qu'il finit souvent par céder aux prières de ses hôtes. C'est le cas, par exemple, avec le bouvier qu'il rencontre quand il s'enfuit de Monbrun et avec qui il accepte de manger, mettant ainsi fin au jeûne qui durait depuis le début de sa quête; plus tard, il cède à l'invitation d'Augier d'Essart. Ici l'incohérence du personnage est encore plus comique, parce qu'il change d'avis en l'espace d'une quinzaine de vers:

Qe per beure ni per manjar,

Dis el, ni per nuil autre far

No remanra c'ades no an. [...]

E dis: A manjar m'er, so cre.

(v. 4611-4613 et 4628)

13. Le caractère parodique de ce passage a été souligné par Limentani, op. cit., p. 95-99, qui l'a mis en relation avec l'épisode de la rêverie dans le Perceval de Chrétien de Troyes, où le héros ne veut pas être dérangé pendant son observation des gouttes de sang sur la neige qui lui rappellent Blanchefleur. 
Ni pour boire, ni pour manger, dit-il, ni pour quoi que ce soit, je ne laisserai de partir à l'instant. [...] Il faudra bien, dit-il, que je mange, à ce que je crois.

Il est indubitable qu'une des scènes clés pour la construction du personnage de Jaufré, pour son devenir homme et adulte, est celle de la confrontation avec le géant lépreux, à laquelle nous avons déjà fait allusion. L'épisode des lépreux est placé au milieu de la première moitié du roman ${ }^{14}$. Ayant croisé l'écuyer d'un chevalier tué par un géant lépreux, Jaufré se met à la recherche de celui-ci et de la jeune fille qu'il a enlevée; sur la route, il rencontre une mère désespérée parce qu'un autre lépreux a enlevé son enfant (lépreux qui est une sorte de serviteur du premier, pour lequel il doit tuer des enfants, le sang des innocents étant censé guérir de la lèpre $\left.{ }^{15}\right)$. Le héros s'introduit dans la maison enchantée où habitent ces lépreux et voit le géant qui s'apprête à violer la jeune fille. Suit un terrible combat d'où Jaufré sort vainqueur, mais, on l'a vu, très éprouvé. Grâce aux indications de l'autre lépreux, Jaufré peut enfin rompre l'enchantement qui l'empêche de sortir de la maison.

Le caractère symbolique d'initiation sexuelle du combat contre le lépreux (v. 2350-2442) a été souligné par Marc-René Jung: la lèpre est traditionnellement une figure de la luxure, le lépreux est un géant, son arme est une massue, Jaufré atteint son ennemi surtout à la partie inférieure du corps ${ }^{16}$. De plus, le héros saigne du nez et de la bouche (v. 2416-2417 et 2446-2447) ; ce fait serait moins étonnant si on le retrouvait dans d'autres combats du roman, mais on ne le rencontre qu'ici et dans l'autre combat contre un géant, le frère de celui-ci: ce dernier, toutefois, frappe Jaufré de son poing et non pas d'une massue (v. 5733-5739). On ne trouve pas non plus de saignement du nez ou de la bouche dans l'archétype médiéval des combats contre les géants : l'affrontement entre Arthur et le géant du Mont-Saint-Michel relaté par Geoffroy de Monmouth ${ }^{17}$. Étant donné que « tout l'épisode [...] est placé sous le signe de la sexualité ${ }^{18} »$, il n'est pas impossible qu'à un niveau

14. V. $2180^{1}-3016$. Le deuxième hommage au roi d'Aragon est placé au milieu de cet épisode (v. 2565-2630).

15. Sur ce motif, ainsi que pour une analyse de cet épisode dans une perspective réaliste, voir P. Rémy, «La Lèpre, thème littéraire au Moyen Âge. Commentaire d'un passage du roman provençal de Jaufré », Le Moyen Âge. Revue d'histoire et de philologie, n 52 , 1946, p. $195-242$.

16. M.-R. Jung, « Lecture de Jaufre », dans Mélanges de langues et de littératures romanes offerts à Carl Theodor Gossen, dir. G. Colón et R. Kopp, 2 vol., Berne, Francke et Liège, Marche Romane, 1976, vol. I, p. 427-451 (surtout p. 441-442).

17. Voir The "Historia Regum Britannie» of Geoffrey of Monmouth. I, Bern, Burgerbibliothek, MS. 568, éd. N. Wright, Cambridge, Brewer, 1985, § 165 ; cf. le passage correspondant dans le Roman de Brut de Wace: La Partie arthurienne du « Brut », éd. I. D. O. Arnold et M. M. Pelan, Paris, Klincksieck, 1962, v. 2937-3010.

18. Jung, art. cit., p. 441. 
inconscient, le saignement du nez et de la bouche renvoie aux effets redoutés d'une castration, voire d'une pénétration: celles-ci auraient été évitées grâce au combat ${ }^{19}$, et la victoire dans cette lutte sanctionne l'entrée de Jaufré dans l'âge viril ${ }^{20}$. Sorte de rite d'initiation, le combat est une performance, à la suite de laquelle Jaufré devient homme. Nous rappellerons, avec Judith Butler, que « l'idée que le genre est performatif a été conçue pour montrer que ce que nous voyons dans le genre comme une essence intérieure est fabriqué à travers une série ininterrompue d'actes ${ }^{21} \gg$.

Bien que par la suite Jaufré puisse être considéré comme un vrai chevalier, on rencontre encore des passages où ses vertus chevaleresques sont, pour ainsi dire, mitigées, au moins au niveau du langage. Le combat contre Taulat de Rougemont nous surprend, les commentateurs l'ont remarqué depuis longtemps : alors que quelques-uns des combats qui le précèdent sont décrits de façon très articulée, celui-ci, qui devrait pourtant être l'un des moments clés du roman, est expédié en une trentaine de vers (6036-6065). Les interprètes ont expliqué que l'auteur voulait montrer que Jaufré remporte facilement la victoire parce qu'il est du côté des justes, et que Taulat est puni à cause de son orgueil ${ }^{22}$. Ce sont les mots du héros lui-même qui autorisent de telles affirmations; ce qui est plus surprenant, c'est que, tout en soulignant l'orgueil de son adversaire, Jaufré rabaisse sa propre valeur:

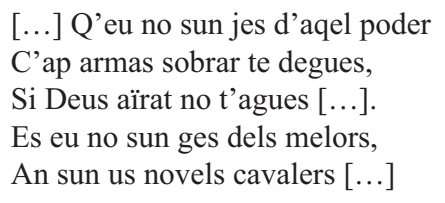

(v. 6084-6113)

19. Sur la corrélation entre l'épistaxis et les organes génitaux, voir W. Fliess, Les Relations entre le nez et les organes génitaux féminins présentées selon leurs significations biologiques, trad. P. Ach et J. Guir, Paris, Le Seuil, 1977 (éd. originale allemande 1897), ch. VI, où l'auteur met en relation le saignement du nez également avec la sexualité masculine; S. Freud, Introduction à la psychanalyse, trad. S. Jankélévitch, Payot, coll. «Petite Bibliothèque Payot », $1961^{2}$ (éd. originale allemande 1916-1917), où on lit que la chute ou l'extraction d'une dent (qui provoque un saignement de la bouche) « signifie certainement la castration, envisagée comme une punition pour les pratiques contre nature » (p. 142). Pour une interprétation d'un saignement du nez comme symbole d'une défloration dans un roman médiéval, dans ce cas incestueuse et d'une jeune fille, voir Le Roman d'Apollonius de Tyr. Version française du XV siècle de l'Histoire d'Apollonius de Tyr, éd. et trad. M. Zink, Paris, UGE, coll. « Livre de poche/Lettres gothiques », 2006 ( $1^{\text {re }}$ éd. 1982), p. 14-15.

20. Sur cet aspect des combats contre les géants, voir J. J. Cohen, Of Giants : Sex, Monsters, and the Middle Ages, Minneapolis, University of Minnesota Press, 1999, surtout p. 73-74. 21. J. Butler, Trouble dans le genre: pour un féminisme de la subversion, trad. C. Kraus, Paris, La Découverte, 2005, "Introduction» à la $2^{\mathrm{e}}$ éd. américaine, 1999, p. 36 ( $1^{\text {re }}$ éd. américaine 1990).

22. Jauss, art. cit., p. 39. 
[...] que je ne suis pas d'une si grande force que je dusse te vaincre avec les armes, si Dieu ne t'avait pas pris en haine [...]. Et je ne suis pas des meilleurs, mais je suis tout nouveau chevalier [...]

À la lumière de ce passage, on pourrait penser que des jugements comme ceux que Jaufré portera sur lui-même au moment de ses tourments amoureux au sujet de Brunissen, en s'accusant de lui être inférieur et de ne pas la mériter (v. 7429-7432), ne sont pas de simples réflexions stéréotypées, quoique de telles expressions apparaissent ailleurs dans la littérature romanesque et dans la lyrique ${ }^{23}$. Peut-être ne faut-il pas donner trop d'importance à toutes ces affirmations de Jaufré, qui témoignent surtout de sa modestie : toutefois, quand Taulat fera son mea culpa devant la reine, bien qu'il qualifie Jaufré de « tut le melhor », il soulignera davantage ses qualités morales que ses vertus chevaleresques (v. 6510-6514) ${ }^{24}$.

$\mathrm{Si}$, dans les romans chevaleresques, l'équivalence entre chevalier et homme joue, les comportements « déplacés » de Jaufré - par rapport à une masculinité monolithique - semblent mettre en récit le truisme, pourtant souvent d'acceptation difficile, selon lequel la virilité définie par les systèmes sociaux réglés par l'hétérosexualité obligatoire ${ }^{25}$ (c'est-à-dire par tous les systèmes sociaux connus) ne s'inscrit pas nécessairement dans une organisation binaire et parallèle opposée à la féminité de façon immuable.

\section{Le personnage de Brunissen}

Les traits de Jaufré qui, sans qu'on puisse les qualifier de "féminins », sont du moins non virils selon une idée essentialiste de la virilité, ont un rapport avec la spécificité du personnage féminin principal, Brunissen. Châtelaine de Monbrun, maîtresse de nombre d'autres châteaux puisqu'elle n'a plus de parents vivants (v. 3059 sq.), elle est présentée sous des traits despotiques, de sorte que, par exemple, elle s'adresse souvent rudement à ses subordonnés (voir, entre autres, ses menaces contre le sénéchal aux v. 7047-7058). Le personnage féminin est donc socialement supérieur au héros masculin: cela n'est pas un fait nouveau dans les romans arthuriens ${ }^{26}$; si les exploits guerriers permettent aux hommes d'acquérir la

23. On lit des propos semblables encore aux vers 8234-8241.

24. Au vers 6519 il dira que Jaufré « Mot es francs et humilz et fins » (« est très franc et modeste et loyal »), et aux v. 6531-6532 il ajoutera qu'il a beaucoup « de bonesa,/ D'umilitat e de franquesa » (« de bonté, de modestie et de franchise »).

25. L'expression « hétérosexualité obligatoire » a été introduite par G. Rubin, L'Économie politique du sexe: transactions sur les femmes et systèmes de sexe/genre, trad. N.-C. Mathieu, Cahiers du CEDREF, 7, 1998; "The Traffic in Women: Notes on the "Political Economy" of Sex », dans Toward an Anthropology of Women, dir. R. R. Reiter, New York et Londres, Monthly Review Press, 1975, p. 157-210.

26. Voir toutefois l'archétype du genre, Érec et Énide, où l'héroïne est la fille d'un vavas- 
gloire, les femmes - qui tombent amoureuses des chevaliers grâce à leur valeur guerrière et à leur beauté, alors qu'elles n'ont souvent pour elles que cette dernière qualité et une vague courtoisie - sont elles-mêmes un instrument pour leur permettre de monter l'échelle sociale. Mais en plus d'être socialement et économiquement supérieure à son amoureux (supériorité dont Jaufré, comme nous l'avons dit, a conscience), Brunissen, comme la plus célèbre Nicolette ${ }^{27}$, possède un tempérament volontaire.

Nous avons vu que, lors de sa première rencontre avec Jaufré, avant de tomber amoureuse de lui, elle voulait le mettre à mort. Plus tard, quand ils se seront avoué réciproquement leur amour, bien qu'il reste secret aux yeux des autres, Brunissen se comportera comme une fiancée qui ose même prendre des décisions à la place de son amoureux: lorsque la fée de Gibel vient demander l'aide de Jaufré au moment où lui et Brunissen se mettent en route pour aller à la rencontre de Mélian de Montmélior, celle-ci répond à la fée la première, bien qu' " entre ssas dentz » (précision qui nous paraît humoristique) :

Piucella, ben parlat en fol,
Car, qui per forsa nu. $1 \mathrm{mi}$ tol,
N'aurai ieu tot so que $\cdot \mathrm{m}$ desir
Enanz que 1 lais de mi partir.

(v. 8099-8102)

Pucelle, vous parlez comme une sotte, car, à moins qu'on ne me l'enlève par force, j'en aurai tout ce que je désire, avant que je le laisse s'éloigner de moi.

Jaufré refuse à son tour de défendre la fée pour l'instant, parce que ses affaires sont trop pressées et importantes (v. 8113-8114). Commence ici sa courte période de "recreantise », l'attitude du chevalier trop amoureux qui délaisse la chevalerie pour rester à côté de la femme qu'il aime (thème rendu célèbre par Érec et Énide); Jaufré sera bientôt contraint de retourner à ses devoirs de chevalier parce qu'il sera enlevé par la fée, qu'il délivrera de la menace de Félon d'Auberue. Encore à la fin du roman, lorsque cette même fée remet à son sauveur des dons enchantés, mais qu'elle veut préalablement « demander une faveur [à Jaufré] » (v. 10515), Brunissen répond la première que ce serait une folie de la lui refuser (v. 10521-10522). De plus, à plusieurs reprises, le narrateur met en scène

seur, alors que le héros est le fils du roi Lac.

27. Dans Aucassin et Nicolette on assiste toutefois à une sorte d'inversion des rôles traditionnels, puisque Aucassin est présenté comme indolent et passif, ce qui n'est pas le cas de Jaufré. Beaucoup d'interprètes ont d'ailleurs vu dans la « chantefable » une œuvre parodique: voir la préface par J. Dufournet de son éd. et trad., Paris, Garnier-Flammarion, 1973, p. 19-34. 
Brunissen avec les personnages masculins, presque sur un pied d'égalité (v. 9511-9513 et 10336-10338) ${ }^{28}$.

La condition privilégiée de Brunissen n'empêche pas qu'elle soit soumise à la volonté de son suzerain, Mélian de Montmélior: si le vassal était soumis au suzerain, à plus forte raison l'était une femme dans la position de vassal. La société médiévale était fondée sur le système de l'échange des femmes pour créer des liens entre les hommes, système typique des sociétés traditionnelles; l'échange des femmes était d'ailleurs central dans la formation des hiérarchies masculines à l'intérieur de la féodalité telle qu'elle est représentée dans le roman chevaleresque ${ }^{29}$. Mais, en ce qui concerne Brunissen, cette soumission est seulement apparente. Les deux amants ont tenu caché leur amour et leurs engagements, et Jaufré doit faire semblant de pousser Mélian à lui « donner » Brunissen; celui-ci promet qu'il la lui «fer[a] dar» (v. 8244). Brunissen fait semblant à son tour de se soumettre à la volonté de Mélian, quand celui-ci lui impose d'épouser Jaufré: « vostra sui tant can viurai », dit-elle (« je suis vôtre tant que je vivrai » [v. 8274]). Le dialogue entre Brunissen et Mélian, toutefois, fait sourire le lecteur informé, et le narrateur n'avait pas négligé de définir l'héroïne, avant sa réponse, comme « cela qui ab sen » (« celle qui est intelligente, maligne » [v. 8268]). Le système de sexe/genre ${ }^{30}$ de l'échange des femmes reste donc en place dans son apparence extérieure: Jaufré avait libéré Mélian de la captivité de Taulat; Mélian, pour lui montrer sa gratitude, lui offre Brunissen; par conséquent, les liens homosociaux entre les deux hommes sont accrus ${ }^{31}$. Le système est en réalité faussé par le fait que Jaufré n'a pas été imposé à Brunissen, mais qu'il a été choisi par elle. De plus, Jaufré n'améliore pas réellement sa situation économique grâce au mariage, du moins si l'on se base sur les vers qui suivent la déclaration

28. À la lumière de cette analyse, peut-être pourrait-on voir sous un angle différent le fait que Brunissen, après s'être dit qu'il revient à l'homme de supplier et à la femme d'écouter (v. 7527 sq.), pousse explicitement Jaufré à déclarer son amour (v. 7736 sq.). Mais le monologue d'une femme qui se dit qu'il serait déshonorant de se déclarer la première, mais qui finit par le faire, est un topos dans les romans médiévaux à partir du Roman d'Eneas, v. 8761 sq. (éd. et trad. A. Petit, Paris, UGE, coll. «Livre de poche/Lettres gothiques », 1997) ; plus précisément, la démarche de Brunissen est proche de celle de la Fénice de Chrétien de Troyes (Cligès, éd. cit., v. 5106 sq.).

29. Le système de l'échange des femmes a été décrit par C. Lévi-Strauss, Les Structures

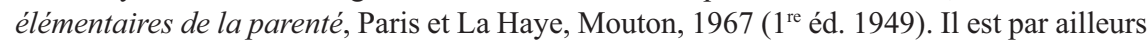
connu que la théorie de Lévi-Strauss, qui définit les femmes comme des « signes », prête à des critiques: pour une relecture sous un angle féministe, voir Rubin, op. cit., et M. Wittig, La Pensée straight, Paris, Balland, 2001, réimpr. Paris, Amsterdam, 2007, surtout les essais III-IV. Sur la formation des hiérarchies dans les romans chevaleresques, voir Gaunt, op. cit., p. 73-74.

30. L'expression « système de sexe/genre » a été introduite par Rubin, op. cit.

31. Sur le concept d'homosocialité, on verra E. K. Sedgwick, Between Men: English Literature and Male Homosocial Desire, New York, Columbia University Press, 1985, p. 1-5. 
d'amour et la promesse de mariage et dans lesquels il affirme qu'il défendra les terres de Brunissen, sans en devenir le maître (v. 7954-7966) ${ }^{32}$.

\section{Conclusion}

Nous nous sommes intéressé aux deux personnages principaux du Jaufré, et surtout au protagoniste masculin. Jaufré a peur, il lui arrive de ne pas maintenir des engagements, de fuir devant des adversaires, de se déprécier; il surmonte une épreuve à haute signification symbolique, le combat contre le lépreux, qui lui permet d'entrer dans l'âge adulte et de devenir un vrai chevalier, mais encore par la suite il se laisse aller à des faiblesses. En revanche, Brunissen se montre très dure avec ses serviteurs, elle aime à prendre des décisions et quelquefois devance les hommes, qui, dans certaines circonstances, semblent l'accueillir dans leur cercle; même quand elle semble céder à leur volonté (Mélian), nous savons que c'est elle qui contrôle la situation.

L'atténuation des caractères traditionnellement attribués aux genres sexués est un des indices qui permettent d'apercevoir dans le roman de Jaufré l'ironie du narrateur: ce n'est pas le seul, et peut-être n'est-ce pas non plus le principal, mais il est légitime de lui attribuer la première place dans une étude critique qui veut mettre en lumière le rôle important du caractère genré des rapports entre les êtres humains dans la détermination des sociétés et de leurs produits culturels. Tout comme les organisations économiques, les systèmes de sexe/genre évoluent avec le temps, et avec ceux-ci les produits culturels qui en sont l'expression. Simon Gaunt a suggéré que, dans les chansons de geste, la personnalité des héros se construit uniquement en rapport avec d'autres personnages masculins. Les femmes sont exclues d'un processus où les hommes sont unis entre eux par des rapports homosociaux (voire de désir homosocial) ${ }^{33}$. Aussi le roman serait-il l'expression d'une société nouvelle: il ne s'agit pas d'une société où les femmes ont acquis une plus grande importance; ce qui a changé, c'est le regard des hommes sur eux-mêmes. On passe d'une société où l'être humain se définit collectivement à une société qui met l'individu au centre de ses intérêts. Cet individu, dans un système patriarcal régi par l'hétéro-

32. Il est vrai que ce point n'est pas tout à fait clair : quand Mélian offrira à Jaufré Brunissen avec tout ce qu'elle possède (v. 8242-8244), le héros n'aura rien à objecter. Ensuite, Jaufré lui-même dira à la reine que Mélian lui a donné « cette femme avec sa terre » (v. 95999600). Le héros ne pouvait pas accepter la terre d'une femme, mais pouvait-il l'accepter d'un homme, qui était le véritable maître du fief en tant que suzerain? Nous ne croyons pas que l'auteur ait pensé à ces questions de droit féodal, mais il est possible que dans le passage de la déclaration il se soit laissé aller à une envolée lyrique, et dans les autres passages il ait représenté les réelles mœurs de son temps.

33. L'argument de Gaunt est bien sûr plus articulé; sur la chanson de geste, voir op. cit., ch. I, « Monologic masculinity ». 
sexualité obligatoire, est nécessairement l'individu mâle, et son individualité ne peut s'exprimer qu'à travers le rapport avec l'autre, "la femme », celle-ci n'étant pas un individu en elle-même, mais seulement en tant que différente de «l'homme », conçu comme l'universel ${ }^{34}$.

Il faut néanmoins établir des distinctions à l'intérieur de ces catégorisations: si une certaine vision monolithique de l'individu mâle qui doit s'affirmer grâce à « la femme », mais en dépit d'elle, est peut-être valable pour les romans les plus anciens (l'Eneas, par exemple), les romans arthuriens sont, dès leur début (c'est-à-dire dès Chrétien de Troyes), teintés d'une ironie qui atténue le trait qui pourtant les distingue: la mise en récit de l'accomplissement d'un destin individuel ${ }^{35}$. Plus le temps passe, moins les auteurs des romans adhèrent aux idéaux arthuriens, les tournant, petit à petit, en ridicule. Jaufré n'est sans doute pas le seul roman à représenter cette évolution: d'autres romans arthuriens en vers ont un caractère parodique (par exemple, Fergus) ou sont très critiques envers le monde qu'ils représentent $(Y d e r)$. Mais dans le roman occitan, la vision ironico-parodique, qui s'exprime de plusieurs façons ${ }^{36}$, se manifeste aussi par le biais d'une récriture des comportements traditionnellement associés aux genres sexués: l'homme a peur, comme Jaufré; la femme est volontaire, comme Brunissen. Aujourd'hui cela ne nous surprend plus - cela ne devrait plus nous surprendre -, mais si l'on rencontre ailleurs des héroïnes « viriles » dans la littérature médiévale, on ne rencontre pas souvent des hommes « féminins ».

Andrea Valentini Collège de France, assistant de recherche

34. Pour une discussion approfondie (et un dépassement) de l'abondante bibliographie sur la question des femmes comme l'Autre, voir Butler, op. cit., p. 72-74 et passim. Pour une remise en question de la prétendue idéalisation des femmes au Moyen Âge, voir R. H. Bloch, Medieval Misogyny and the Invention of Western Romantic Love, Chicago et Londres, University of Chicago Press, 1991; sur l'amour courtois, on lira le ch. VI, « The love lyric and the paradox of perfection », ainsi que le ch. III de Gaunt, op. cit., «Troubadours, ladies and language: the canso ».

35. Sur l'ironie de Chrétien, voir, entre autres, P. Haidu, Aesthetic Distance in Chrétien de Troyes: Irony and Comedy in "Cligès » and « Perceval », Genève, Droz, 1968.

36. Par exemple, elle fixe certaines règles de façon maniériste: les aventures ne se présentent plus naturellement, elles sont produites. Nous pensons aux épisodes initial et final du chevalier enchanteur qui se transforme respectivement en bête fantastique et en oiseau pour satisfaire la soif d'aventures du roi Arthur: pour une analyse de ces épisodes, voir Schmolke-Hasselmann, op. cit., p. 71-72. 